\title{
Systems Modeling Usage in Project Management among Junior and Senior Business System Developers
}

\author{
Timothy Joshua M. Ventayen ${ }^{1} \&$ Randy Joy M. Ventayen ${ }^{2}$ \\ ${ }^{1}$ Business Administration Program, Pangasinan State University, Bayambang Campus, Philippines \\ ${ }^{2}$ University Web Administrator, Pangasinan State University, Philippines \\ Correspondence: Timothy Joshua M. Ventayen, PSU Bayambang Campus, Bayambang, Pangasinan. Tel: 639- \\ 186-200-902. E-mail: dayjx@yahoo.com
}

Received: March 30, 2018; Accepted: May 18, 2018; Published: May 21, 2018

The research is financed by Pangasinan State University, Lingayen Campus.

\begin{abstract}
Unified Modeling Language (UML) is accepted as the standard for modeling software designs and is commonly used in software development Thus this paper investigated the practice of Junior and Senior Business System Developers in the use of UML and other Diagrams. Part of the study is the UML Systems modeling usage for Junior Developers by investigating the documentation used in development. This study used several methods in gathering data, interview, and questionnaire from junior and senior developers was used to come up with a result. It also investigates the possible usage of UML Diagrams in Business field which will be a basis for a future proposed content curriculum for both Business Administration and Information Technology. The result of the study shows that junior developers are not using UML Systems modeling, while senior developers increase as the experience increase in development. Most of the respondents used a diagram that is outside in the UML family. The study also shows that UML helps the managers and business people to build models and diagram that helps them to put things into perspective. It proposed that UML Diagrams should include in business Subject for both programs, the business and information technology program should unite to increase the UML usage.
\end{abstract}

Keywords: unified modeling language, UML practice and usage

\section{Introduction}

There is a saying that A picture is worth a thousand words (Find It On eBay: Using Pictures Instead of Words. 2017), that is why system modeling and software development methodologies forge a common visual language in the complex world of project development that would also be understandable for business users and anyone who wants to understand a system. This systems modeling helps to unite the business management unit and the software engineering team and will bridge the gap in understanding each other. UML is the de facto standard for modeling software designs and commonly used in software development, it was considered as quality tools used in modeling (Genero et al., 2011). However, there are speculations that little was known about the use of UML in the software project management among Junior developers and Senior developers, and this is the primary purpose of this study.

Based on the practice, there are three basic approaches applied to software development methodology frameworks which are the prototyping, waterfall, and spiral (Reading: Software Development Process | Introduction to Computer Applications and Concepts. 2018). The software developer has a choice on what methodology was used in the development. The approaches are necessary for software developers interpreted the flow of the project to the project managers and business modelers.

The UML diagram emerged in the mid-1990s (Rumbaugh et al., 2004) through the combination of previously competing object-oriented analysis and design (OOAD) approaches. Several studies prove that The UML is an industry standard for object-oriented software engineering (Dobing \& Parsons, 2008; Medvidovic et al., 2002). The version of the UML 2.0 has 13 types of diagrams, divided into three categories: Six diagram types represent static application structure; three represent general types of behavior; and four represent different aspects of interactions, while UML 2.5 specification defines two important kinds of UML diagram: structure diagrams and behavior diagrams. (Fakhroutdinov, 2013) This paper will also evaluate if the developers are aware of the use of the industry standard for the object-oriented software engineering. 
In this study, the researchers defined Junior developers as graduating students taking up computer courses such as BS Information Technology and BS Computer Science. While Senior developers was defined as working professionals and alumni of one University that is currently practicing the profession, the underemployed worker was not considered to be part of the respondents.

\subsection{The Objective of the Study and Statement of the Problem}

The paper investigated the practice of Junior and Senior Business System Developers in the use of UML and other Diagram. There are two parts of this study; the first part is the UML Systems modeling usage for Junior Developers, which sought to answer the following question (1) What are the type of project developed? (2) What diagram used in the project development? What types of UML diagrams used? The second part of the study is an investigation using the interview and questionnaire from junior and senior developers. The result of the study will be a basis for a future proposed content curriculum for both Business Administration and Information Technology.

\subsection{The Significance of the Study}

Business administration and Information technology is a right combination of discipline to achieve the organizational goal of the company. The most organization today has integrated MIS department that caters the needs of information management and business forecasting. The importance of this study is to determine the usage of UML Diagrams that could help the curriculum improve both programs to integrate UML in the subject Project Management and System Analysis and Design. This paper is also an eye-opener to the educational system of the country.

\section{Related Literature and Studies}

A book chapter is written by Mouheb, et al., describes UML as a general-purpose modeling language in the field of software and systems engineering. It was created and standardized by the Object Management Group (OMG) in 1997. The book chapter consist useful information about the introductory part and use of UML diagrams; it helps readers to appreciate the importance of UML (Mouheb et al., 2015). Documentation about UML is also available; there is a reference manual of the UML diagrams (Rumbaugh et al., 2017) which was intended to be a complete and useful reference to the Unified Modeling Language (UML). The reference manual was considered as useful for the developer, architect, project manager, system engineer, programmer, analyst, contracting officer, customer, and anyone else who needs to specific usage. The manual is also suitable for a beginner who is still starting to study UML. As mention by one encyclopedia (Fettke, 2005) that UML enables practitioners to collaborate and also contribute to the development of the whole system, this is evident because UML is assumed to bridge the gap between the different fields such as the Business Managers and IT Experts. In the study conducted by Reggio, et al. (2014) presented result about the knowledge and usage of the UML using opinion survey with 275 participants from both industry and academia. The study also analyzed the influence of different factors such as the working environment, role, seniority, education, and gender. In their study, the results show that the level of knowledge and usage of the various types of diagrams is quite different and that these differences are quite stable also when considering different categories. Truly UML is described as "the lingua franca" of software engineering (Petre, 2013).

\section{Methodology}

The research design of this study is quantitative and qualitative descriptive approach. It is a combination of data gathering from documents, survey questionnaire, an interview. This paper gathers information from the project study of senior year students in one state university. The researcher evaluated the usage of UML diagrams used based on the information available in the thesis of each student in the library. Our findings also include the different diagram that is useful in software development and not limited to UML diagrams. Non-Probability sampling was adopted, purposive and convenience sampling was used to determine the respondents. For the data gathering of this study in the first part, the proponent will investigate the thesis of the students in the library in two programs, the Information Technology and Computer Science.

For the second part, the researcher will float a questionnaire to the junior developers and interview graduate alumni of a university. The information of alumni will be gathered thru the office of the guidance counselor and check the IT practitioners.

A Competencies Proficiency Scale (Competencies Proficiency Scale) was used in the questionnaire to determine the level of proficiency of the developers. A Frequency and percentage used in every level of proficiency and an average weighted mean for the overall result were adopted to determine the overall proficiency of the developers. A graphical comparison in a spreadsheet software was also used to determine the graphical relationship between the level of competency for both junior and senior developers. 
Table 1. Competencies Proficiency Scale

\begin{tabular}{lll}
\hline Scale & Equivalent & Mean Scale \\
\hline 1 & Fundamental Awareness (basic knowledge) & $1.00-1.50$ \\
2 & Novice (limited experience) & $1.51-2.50$ \\
3 & Intermediate (practical application) & $2.51-3.50$ \\
4 & Advanced (applied theory) & $3.51-4.50$ \\
5 & Expert (recognized authority) & $4.51-5.00$ \\
\hline
\end{tabular}

\section{Results}

The results of the study show the usage of diagrams in the thesis of junior developers from 2013 to 2016, there are 8 Computer Science and 27 Information Technology investigated researchers. For the questionnaire floated, there are 97 junior developers in the SY 2017-2018, and 18 senior developers interviewed before floating the questionnaire and graduated during 2012 to 2015 from the same institution as a respondent for the data gathering.

\subsection{Software Project Developed and Methodologies}

In the case of the junior developers, with a total of 35 total number of research papers. 15 papers develop a mobile application. 12 papers documented the creation of a website, while 8 created system application and web application. For the methodology used, mobile application developers use Scrum Methodology and RAD methodology for website development and application development.

\subsection{Diagram used in Documenting Project}

Based on the findings, the majority of the junior developers are not aware of the usage of the UML diagrams. As shown in the table below, most of the UML Diagram not used in the development. Other diagrams that are not part of the UML family used are italic in the table. Use Case, Activity Diagram and Sequence Diagram are the only UML diagram used in the documentation of the junior developers.

Table 2. Use of UML and other Diagram in Thesis of Junior Developers

\begin{tabular}{llllll}
\hline Diagram & & \multicolumn{3}{l}{ Junior } & Total \\
\hline UML & & CS & IT & Total & Percentage \\
\hline & Class Diagram & 0 & 0 & 0 & $0 \%$ \\
& Object Diagram & 0 & 0 & 0 & $0 \%$ \\
& Package Diagram & 0 & 0 & 0 & $0 \%$ \\
& Composite Structure Diagram & 0 & 0 & 0 & $0 \%$ \\
& Component Diagram & 0 & 0 & 0 & $0 \%$ \\
& Deployment Diagram & 0 & 0 & 0 & $0 \%$ \\
Profile Diagram & 0 & 0 & 0 & $0 \%$ \\
& Use Case Diagram & 1 & 2 & 3 & $9 \%$ \\
& Information flow Diagram & 0 & 0 & 0 & $0 \%$ \\
& Activity Diagram & 0 & 1 & 1 & $3 \%$ \\
& State Machine Diagram & 0 & 0 & 0 & $0 \%$ \\
& Interaction Diagram & 0 & 0 & 0 & $0 \%$ \\
& Sequence Diagram & 1 & 1 & 2 & $6 \%$ \\
& Communication Diagram & 0 & 0 & 0 & $0 \%$ \\
& Timing Diagram & 0 & 0 & 0 & $0 \%$ \\
Interaction Overview Diagram & 0 & 0 & 0 & $0 \%$ \\
\hline Other & & & & & \\
\hline & Flowchart & 8 & 27 & 35 & $100 \%$ \\
& Data Flow Diagram & 6 & 19 & 25 & $71 \%$ \\
Entity Relation Diagram & 7 & 16 & 23 & $66 \%$ \\
Gantt Chart & 8 & 23 & 31 & $89 \%$ \\
\hline
\end{tabular}

The table shows that majority of the Junior Developers use flowchart in the development process of the project, followed by the Gantt Chart, DFD, and ERD. The flowchart is the most commonly used diagram by the junior developers which 100 percent of them is using it. 


\subsection{Diagrams used by Junior Developers}

After investigating the documents of the junior developers, a questionnaire was created containing the UML diagrams and other diagram used. On the result of the study regarding the level of competency in the different UML diagrams, it is visible that only 3 diagram under the UML family mastered.

Table 3. Use of UML and other Diagram of Junior Developers

\begin{tabular}{|c|c|c|c|c|c|c|c|c|c|c|c|}
\hline & & 1 & & 2 & & 3 & & 4 & & 5 & AVM \\
\hline Class Diagram & 43 & $44.33 \%$ & 25 & $25.77 \%$ & 13 & $13.40 \%$ & 12 & $12.37 \%$ & 4 & $4.12 \%$ & 2.06 \\
\hline Object Diagram & 32 & $32.99 \%$ & 36 & $37.11 \%$ & 28 & $28.87 \%$ & 1 & $1.03 \%$ & 0 & $0.00 \%$ & 1.98 \\
\hline Package Diagram & 34 & $35.05 \%$ & 24 & $24.74 \%$ & 32 & $32.99 \%$ & 5 & $5.15 \%$ & 2 & $2.06 \%$ & 2.14 \\
\hline Composite Structure Diagram & 25 & $25.77 \%$ & 42 & $43.30 \%$ & 23 & $23.71 \%$ & 3 & $3.09 \%$ & 4 & $4.12 \%$ & 2.16 \\
\hline Component Diagram & 42 & $43.30 \%$ & 36 & $37.11 \%$ & 18 & $18.56 \%$ & 1 & $1.03 \%$ & 0 & $0.00 \%$ & 1.77 \\
\hline Deployment Diagram & 23 & $23.71 \%$ & 47 & $48.45 \%$ & 9 & $9.28 \%$ & 15 & $15.46 \%$ & 3 & $3.09 \%$ & 2.26 \\
\hline Profile Diagram & 41 & $42.27 \%$ & 24 & $24.74 \%$ & 31 & $31.96 \%$ & 1 & $1.03 \%$ & 0 & $0.00 \%$ & 1.92 \\
\hline Use Case Diagram & 1 & $1.03 \%$ & 4 & $4.12 \%$ & 7 & $7.22 \%$ & 21 & $21.65 \%$ & 64 & $65.98 \%$ & 4.47 \\
\hline Information flow Diagram & 31 & $31.96 \%$ & 34 & $35.05 \%$ & 21 & $21.65 \%$ & 8 & $8.25 \%$ & 3 & $3.09 \%$ & 2.15 \\
\hline Activity Diagram & 3 & $3.09 \%$ & 4 & $4.12 \%$ & 17 & $17.53 \%$ & 26 & $26.80 \%$ & 47 & $48.45 \%$ & 4.13 \\
\hline State Machine Diagram & 55 & $56.70 \%$ & 27 & $27.84 \%$ & 15 & $15.46 \%$ & 0 & $0.00 \%$ & 0 & $0.00 \%$ & 1.59 \\
\hline Interaction Diagram & 34 & $35.05 \%$ & 21 & $21.65 \%$ & 16 & $16.49 \%$ & 17 & $17.53 \%$ & 9 & $9.28 \%$ & 2.44 \\
\hline Sequence Diagram & 1 & $1.03 \%$ & 2 & $2.06 \%$ & 7 & $7.22 \%$ & 29 & $29.90 \%$ & 58 & $59.79 \%$ & 4.45 \\
\hline Communication Diagram & 43 & $44.33 \%$ & 26 & $26.80 \%$ & 12 & $12.37 \%$ & 10 & $10.31 \%$ & 6 & $6.19 \%$ & 2.07 \\
\hline Timing Diagram & 48 & $49.48 \%$ & 24 & $24.74 \%$ & 20 & $20.62 \%$ & 4 & $4.12 \%$ & 1 & $1.03 \%$ & 1.82 \\
\hline Interaction Overview Diagram & 18 & $18.56 \%$ & 32 & $32.99 \%$ & 32 & $32.99 \%$ & 10 & $10.31 \%$ & 5 & $5.15 \%$ & 2.51 \\
\hline Flowchart & 0 & $0.00 \%$ & 1 & $1.03 \%$ & 17 & $17.53 \%$ & 16 & $16.49 \%$ & 63 & $64.95 \%$ & 4.45 \\
\hline Data Flow Diagram & 1 & $1.00 \%$ & 5 & $5.15 \%$ & 14 & $14.43 \%$ & 32 & $32.99 \%$ & 45 & $46.39 \%$ & 4.19 \\
\hline Entity Relation Diagram & 0 & $0.00 \%$ & 1 & $1.03 \%$ & 20 & $20.62 \%$ & 18 & $18.56 \%$ & 58 & $59.79 \%$ & 4.37 \\
\hline \multirow[t]{2}{*}{ Gantt Chart } & 1 & $1.03 \%$ & 1 & $1.03 \%$ & 8 & $8.25 \%$ & 23 & $23.71 \%$ & 64 & $65.98 \%$ & 4.53 \\
\hline & & & & & & & & & & & 2.87 \\
\hline
\end{tabular}

Based on the result of the survey, the junior developers are not much aware and competent in the use of UML Diagrams. Junior Developers average weighted mean on the competency proficiency is 2.50 with a descriptive equivalent of Novice.

\subsection{Diagram used by Senior Developers}

The same questionnaire was given to the senior developers for data gathering procedure. Based on the result of the survey, there is visibility on the mastery of several UML Diagrams compared to the junior developers. While Sequence, Use Case, and Activity Diagrams are the most mastered diagram, it was shown in the tabulated result that there is no single UML Diagram that is unknown. It is an indicator that senior developers encounter several UML Diagrams along the way.

Based on the result of the survey, the senior developers are not much aware and competent in the use of UML Diagrams, but there is an increase of competency compared to the junior developers with an average weighted mean on the competency proficiency of 2.90 with a descriptive equivalent of Intermediate.

Based on the interviews from the Senior Developers, most of the 18 senior developers experience in development of a website using CMS WordPress, while the majority of them has experience in PHP coding, most of them did not use manual coding to build a website. Four senior developers have experience in contributing to application development in both software application and mobile development. Most of the senior developers use UML diagrams that they met from the workplace. 
Table 4. Use of UML and other Diagram of Senior Developers

\begin{tabular}{llllllllllll}
\hline & 1 & & 2 & & 3 & & 4 & & 5 & & AVM \\
\hline Class Diagram & 6 & $33.33 \%$ & 3 & $16.67 \%$ & 4 & $22.22 \%$ & 3 & $16.67 \%$ & 2 & $11.11 \%$ & 2.56 \\
Object Diagram & 8 & $44.44 \%$ & 3 & $16.67 \%$ & 3 & $16.67 \%$ & 1 & $5.56 \%$ & 3 & $16.67 \%$ & 2.33 \\
Package Diagram & 5 & $27.78 \%$ & 3 & $16.67 \%$ & 7 & $38.89 \%$ & 2 & $11.11 \%$ & 1 & $5.56 \%$ & 2.50 \\
Composite Structure Diagram & 6 & $33.33 \%$ & 1 & $5.56 \%$ & 4 & $22.22 \%$ & 3 & $16.67 \%$ & 4 & $22.22 \%$ & 2.89 \\
Component Diagram & 2 & $11.11 \%$ & 5 & $27.78 \%$ & 5 & $27.78 \%$ & 4 & $22.22 \%$ & 2 & $11.11 \%$ & 2.94 \\
Deployment Diagram & 6 & $33.33 \%$ & 4 & $22.22 \%$ & 4 & $22.22 \%$ & 2 & $11.11 \%$ & 2 & $11.11 \%$ & 2.44 \\
Profile Diagram & 4 & $22.22 \%$ & 2 & $11.11 \%$ & 4 & $22.22 \%$ & 6 & $33.33 \%$ & 2 & $11.11 \%$ & 3.00 \\
Use Case Diagram & 0 & $0.00 \%$ & 2 & $11.11 \%$ & 3 & $16.67 \%$ & 3 & $16.67 \%$ & 10 & $55.56 \%$ & 4.17 \\
Information flow Diagram & 1 & $5.56 \%$ & 11 & $61.11 \%$ & 4 & $22.22 \%$ & 1 & $5.56 \%$ & 1 & $5.56 \%$ & 2.44 \\
Activity Diagram & 1 & $5.56 \%$ & 3 & $16.67 \%$ & 1 & $5.56 \%$ & 5 & $27.78 \%$ & 8 & $44.44 \%$ & 3.89 \\
State Machine Diagram & 6 & $33.33 \%$ & 6 & $33.33 \%$ & 2 & $11.11 \%$ & 1 & $5.56 \%$ & 3 & $16.67 \%$ & 2.39 \\
Interaction Diagram & 5 & $27.78 \%$ & 5 & $27.78 \%$ & 1 & $5.56 \%$ & 2 & $11.11 \%$ & 5 & $27.78 \%$ & 2.83 \\
Sequence Diagram & 0 & $0.00 \%$ & 1 & $5.56 \%$ & 1 & $5.56 \%$ & 4 & $22.22 \%$ & 12 & $66.67 \%$ & 4.50 \\
Communication Diagram & 8 & $44.44 \%$ & 7 & $38.89 \%$ & 1 & $5.56 \%$ & 1 & $5.56 \%$ & 1 & $5.56 \%$ & 1.89 \\
Timing Diagram & 4 & $22.22 \%$ & 7 & $38.89 \%$ & 4 & $22.22 \%$ & 1 & $5.56 \%$ & 2 & $11.11 \%$ & 2.44 \\
Interaction Overview Diagram & 6 & $33.33 \%$ & 1 & $5.56 \%$ & 1 & $5.56 \%$ & 5 & $27.78 \%$ & 5 & $27.78 \%$ & 3.11 \\
Flowchart & 0 & $0.00 \%$ & 0 & $0.00 \%$ & 0 & $0.00 \%$ & 1 & $5.56 \%$ & 17 & $94.44 \%$ & 4.94 \\
Data Flow Diagram & 0 & $0.00 \%$ & 0 & $0.00 \%$ & 0 & $0.00 \%$ & 4 & $22.22 \%$ & 14 & $77.78 \%$ & 4.78 \\
Entity Relation Diagram & 0 & $0.00 \%$ & 0 & $0.00 \%$ & 0 & $0.00 \%$ & 2 & $11.11 \%$ & 16 & $88.89 \%$ & 4.89 \\
Gantt Chart & 0 & $0.00 \%$ & 0 & $0.00 \%$ & 0 & $0.00 \%$ & 1 & $5.56 \%$ & 17 & $94.44 \%$ & 4.94 \\
& & & & & & & & & & & 3.29 \\
\hline
\end{tabular}

Table 5. AWM Proficiency of UML and Non-UML

\begin{tabular}{lll}
\hline Diagram & Junior & Senior \\
\hline UML & 2.50 & 2.90 \\
Non-UML & 4.38 & 4.89
\end{tabular}

In the proficiency in UML, junior developers are Novice in the use of most Diagram, while senior developers have an average weighted mean of 2.90 with a descriptive equivalent of Intermediate. On the proficiency in Non-UML, junior developers are Advanced" in the use of the diagram, while the senior developers are "Expert."

\section{Difference Between Junior and Senior}

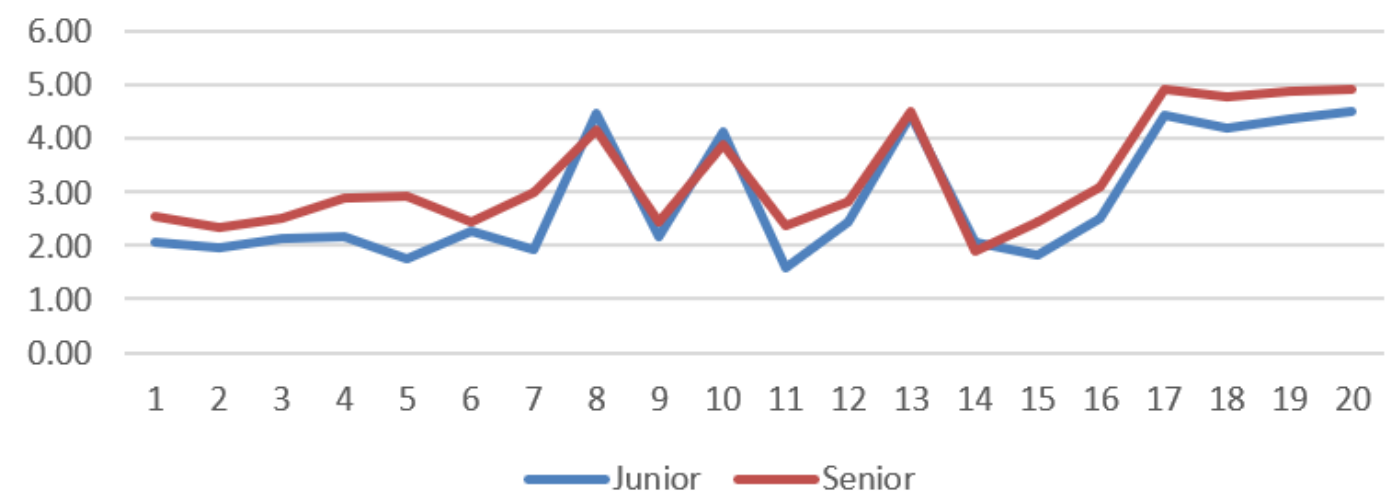

Figure 1. Difference between Junior and Senior Developers in UML Usage

As shown in the figure, there is an increase in the level of competency proficiency in the use of UML. Based on several encountered interview from the junior developers. UML is not being tackled in the subject. While others are being encountered in the subject System Analysis and Design, most of the UML Diagram is new and unheard. On the other side, the senior developers encountered several UML diagram along the way. The search engine was a starting point for them to learn about UML Diagrams. The senior developers also verified that UML Diagram 
was not being taught during the college days. The junior developers encountered most of the non-UML diagram in the Project Management subject in the college and the senior developers also encountered during the college days and proficiency was increased as the number of project increases.

Based on the interview, the senior developers use the UML Diagram to communicate the plan for software development to the stakeholders. According to the senior developers, there is a gap between software engineers and business analyst regarding communication and understanding the requirement of the project, thus the UML diagram and other non-UML diagram bridge the gap. In the result of the interview, we see the vital importance of the UML diagram in business modeling and communication.

\section{UML in Business, A Proposed Intervention}

There is study investigates that UML is not only applicable in System Modeling but also business modeling (Rodríguez et al., 2006). In the study conducted by Ionita, the researcher elaborates weather UML, can be a useful tool in business modeling and administration (Ionita, 2010). The paper analyzed the advantage of modeling language that has to offer, and it is useful too in expressing structure, interaction, and behavior as well. It generally helps the managers and business people to build models and diagram that helps them to put things into perspective.

The researchers propose that UML Diagram should be introduced in both programs, the Business Administration Program and Information Technology. In the Operations Mangement major of BSBA, there are subjects like Project Management; Productivity and Quality Tools; and other technology related subject such as managing Logistics that some system was developed (Jiang et al., 2007). It is proposed that UML Diagram should be included in the syllabus of the subject for future managers to maximize the understanding and contribution of system and project development. While on the side of Computer Science and Information Technology Program, it is proposed that it should be included in the subject System Analysis and Design; Project Management; and other Software Development subject. In this regard, the gap between IT and Business will make a break.

\section{Conclusion and Recommendation}

The result of the study shows that Junior Developers and even Senior Developers are not using most of the UML Diagrams, but using other types of diagram outside the UML. While it was noticed that there are changes in the use of UML diagrams among senior developers after graduation, the majority of the UML diagrams are not being used by the developers itself. Several factors might affect the result such as UML diagram is not included in the syllabus of the student or not being introduced. Senior developers encountered some of the UML diagrams along the way but didn't use another diagram its either not used in the current project or still in the process of discovery. It is recommended that UML diagram should be included in the syllabus in one of the courses in BSIT and BSCS, it is either in System Analysis and Design or programming subjects. UML should not be only exclusively as a language for software developers; more excellent knowledge of UML diagrams in the business field is needed and to the whole organization.

\section{Acknowledgments}

The author would like to give thanks to Dr. Nova Arquillano, the coordinator for Research in PSU Lingayen Campus for allowing us to preset this research study during the Campus Research Forum at PSU Lingayen Campus.

\section{References}

$\begin{array}{lllll}\text { Competencies } & \text { Proficiency } & \text { Scale. } & \text { (n.d.). } & \text { Retrieved }\end{array}$ https://hr.nih.gov/workingnih/competencies/competencies-proficiency-scale

Dobing, B., \& Parsons, J. (2008). Dimensions of UML diagram use: a survey of practitioners. Journal of Database Management, 19(1), 1. https://doi.org/10.4018/jdm.2008010101

Fakhroutdinov, K. (2013, November 25). UML 2.5 Diagrams Overview. Retrieved November 5, 2017, from http://www.uml-diagrams.org/uml-25-diagrams.html

Fettke, P. (2005). Unified modeling language. In Encyclopedia of Information Science and Technology, First Edition (pp. 2921-2928). IGI Global. https://doi.org/10.4018/978-1-59140-553-5.ch520

Find It On eBay: Using Pictures Instead of Words. (2017). Retrieved May 5, 2018, from https://www.ebayinc.com/stories/news/find-it-on-ebay-using-pictures-instead-of-words

Genero, M., Fernández-Saez, A. M., Nelson, H. J., Poels, G., \& Piattini, M. (2011). Research review: A systematic literature review on the quality of UML models. Journal of Database Management (JDM), 22(3), 46-70. https://doi.org/10.4018/jdm.2011070103

Ionita, D. (2010). Uml In Business Administration. Journal of Knowledge Management, Economics and 
Information Technology, 1(1), 1-6. Retrieved from https://ideas.repec.org/a/spp/jkmeit/1128.html

Jiang, S. L., Zheng, Z., Gao, X. Q., \& He, L. M. (2007). Analysis and design of simulation system for logistics in steelmaking process based on uml. Journal of System Simulation, 16, 016.

Medvidovic, N., Rosenblum, D. S., Redmiles, D. F., \& Robbins, J. E. (2002). Modeling software architectures in the Unified Modeling Language. ACM Transactions on Software Engineering and Methodology (TOSEM), 11(1), 2-57. https://doi.org/10.1145/504087.504088

Mouheb, D., Debbabi, M., Pourzandi, M., Wang, L., Nouh, M., Ziarati, R., ... Lima, V. (2015). Unified Modeling Language. In Aspect-Oriented Security Hardening of UML Design Models (pp. 11-22). Springer International Publishing. https://doi.org/10.1007/978-3-319-16106-8_2

Petre, M. (2013, May). UML in practice. In Proceedings of the 2013 International Conference on Software Engineering (pp. 722-731). IEEE Press. https://doi.org/10.1109/ICSE.2013.6606618

Reading: Software Development Process | Introduction to Computer Applications and Concepts. (2018). Retrieved May 5, 2018, from https://courses.lumenlearning.com/zeliite115/chapter/reading-software-developmentprocess/

Reggio, G., Leotta, M., \& Ricca, F. (2014, September). Who knows/uses what of the uml: A personal opinion survey. In International Conference on Model Driven Engineering Languages and Systems (pp. 149-165). Springer, Cham. https://doi.org/10.1007/978-3-319-11653-2_10

Rodríguez, A., Fernández-Medina, E., \& Piattini, M. (2006, September). Towards a UML 2.0 extension for the modeling of security requirements in business processes. In International Conference on Trust, Privacy and Security in Digital Business (pp. 51-61). Springer, Berlin, Heidelberg. https://doi.org/10.1007/11824633_6

Rumbaugh, J., Booch, G., \& Jacobson, I. (2017). The unified modeling language reference manual. Addison Wesley.

Rumbaugh, J., Jacobson, I., \& Booch, G. (2004). Unified modeling language reference manual, the. Pearson Higher Education.

\section{Copyrights}

Copyright for this article is retained by the author(s), with first publication rights granted to the journal.

This is an open-access article distributed under the terms and conditions of the Creative Commons Attribution license (http://creativecommons.org/licenses/by/4.0/). 